\title{
Controle de Suspensão Ativa Automotiva por Lógica Difusa
}

\author{
Neusa V. da Silva* Waldemar Bonventi Jr. ${ }^{* *}$ \\ * Processos Tecnológicos e Ambientais, Universidade de Sorocaba, SP, \\ (e-mail: neusa.silva@uniso.br). \\ ** Processos Tecnológicos e Ambientais, Universidade de Sorocaba, SP, \\ (e-mail: waldemar.bonventi@prof.uniso.br).
}

\begin{abstract}
It was developed a fuzzy controller for active automotive suspension, with parameters refined by simulation. The suspension model used was $1 / 4$ vehicle (bus). The tuning parameters of the PD fuzzy controller were obtained and the result compared to a conventional tuned PD model. As control goal, the settling time was stipulated in less than 5 seconds and less oscilations as possible.

Resumo: Foi desenvolvido o projeto de um controlador fuzzy para suspensão ativa automotiva, com seus parâmetros refinados por simulação. O modelo de suspensão utilizado foi de $1 / 4$ de veículo (ônibus). Foram obtidos os parâmetros de sintonia do controlador PD fuzzy e o resultado comparado com um modelo PD convencional sintonizado. Como meta de controle, foram estipulados acomodação em menos de 5 segundos com menor número de oscilações.
\end{abstract}

Keywords: PD Fuzzy Control; Active Automotive Suspension; Mass-Spring Damped System; 1/4 Car Model; Ziegler-Nichols Method; Jantzen's Equivalent Method.

Palavras-chaves: Controle Fuzzy PD; Suspensão Ativa Automotiva; Sistema Massa-Mola Amortecido; Modelo de 1/4 de Veículo; Método de Ziegler-Nichols; Método de Jantzen.

\section{INTRODUÇÃO}

O sistema de suspensão automotiva isola o veículo das irregularidades do piso e vibrações, para não transferilos aos passageiros e à carga. Consiste em um conjunto amortecimento e restauração em cada roda, ou seja, um sistema massa-mola-amortecido (Halliday et al., 2002). Os objetivos de uma suspensão veicular são: (a) sustentar o peso da carroceria do veículo e proporcionar passeios confortáveis e seguros em vários tipos de superfícies rodoviárias; (b) isolar o corpo do veículo de distúrbios externos provenientes de superfícies irregulares e internos criados por curvas, aceleração ou desaceleração; (c) reagir a variações de carga, geradas por mudanças no número de passageiros e bagagem; (d) manter um contato firme entre a estrada e os pneus, garantindo que as rodas sigam o perfil rodoviário para um bom desempenho e segurança na condução.

As suspensões automotivas são classificadas em três tipos: passivas, semiativas e ativas, de acordo com os seus elementos constituintes e o desempenho fornecido. A suspensão ativa consiste em um atuador que compense os deslocamentos e vibrações da via. Essa é regulada por um algoritmo de controle usando dados de sensores conectados ao veículo. Também é composta por um atuador e uma mola mecânica, ou ambos mais um amortecedor. A mola suporta a carga estática da massa suspensa e o atuador de força fornece a força reativa necessária para minimizar ou absorver a deformação causada por irregularidades do solo. O atuador pode ser hidráulico, pneumático ou ele- tromagnético, ou ainda uma solução híbrida (Mulla and Unaume, 2013).

Com um controlador proporcional-integral-diferencial (PID) atuando entre a suspensão e a carroceria, Popovic et al. (2000) obtiveram, para os casos de um automóvel e um ônibus, um sobressinal de cerca de $5 \%$, e tempo de estabilização de aproximadamente $2,5 \mathrm{~s}$, sendo a frequência natural do sistema cerca de $1 \mathrm{~Hz}$. Pontelli (2012) estudou o controle das oscilações verticais da barra que sustenta os bicos pulverizadores de um equipamento agrícola (suspensão trapezoidal - quatro barras), com simulações em que a frequência imposta pelas oscilações do terreno variou de 0,1 a 2,0 Hz. Foram simulados controle PID e difuso, com oscilações tipo degrau, harmônica e randômica, não havendo grandes diferenças entre elas, exceto na oscilação randômica. Nesta, o controlador difuso apresentou um desempenho melhor. Em geral, as suspensões ativas hidráulicas ou pneumáticas são adequadas para aplicações de baixa largura de banda (de frações a poucos Hz). Corriga et al. (1991) estudaram a otimização da constante elástica da mola e do coeficiente do amortecedor na suspensão hidráulica ativa para minimizar a potência necessária para sua operação. Strassberger and Guldner (2004) descreveram o sistema de barra estabilizadora ativa desenvolvido pela BMW, incorporado à suspensão hidráulica ativa. Outro exemplo comercial é dado pelo sistema de controle de corpo ativo (ABC - Active Body Control) da MercedesBenz. Este sistema também abaixa lentamente o veículo a velocidades mais altas (Daimler, 2016). A regeneração de energia do sistema de suspensão ativa eletromagnética foi investigada por Soleymani (2010), em veículos elétricos 
híbridos com base nos algoritmos de simulação desenvolvidos.

Na simulação de um sistema de suspensão, utilizamse os modelos full car (apoio nas quatro rodas), half car (suspensão dianteira ou traseira) e de $1 / 4$ de veículo (apenas um conjunto roda-amortecedor) (Mulla and Unaume, 2013). Empregamos aqui o modelo 1/4 (figura 1), reduzindo-o a um sistema massa-mola paralelo acoplado. Este apresenta dois graus de liberdade, os demais, acrescentam modos de vibração transmitidos pela rigidez da carroceria. O estudo do modelo $1 / 4$ mostra o comportamento mais evidente da suspensão (em primeira ordem). Senthilkumar et al. (2018) desenvolveram um controlador fuzzy para o modelo full car acoplando quatro sistemas em paralelo e considerando a carroceria totalmente rígida. Changizi and Rouhani (2011) apresentaram um trabalho bastante similar ao realizado aqui, mas em geral, quando o controlador fuzzy diminuia o pico da oscilação, aumentava o tempo de acomodação. Al-Mutar and Abdalla (2015) realizaram uma sintonia fina nas funções de pertinência usando Particle Swarm Optimization reduzindo muito o pico de oscilação, mas os parâmetros físicos da suspensão estudada foram bem diferentes dos utilizados aqui. Em todas as simulações apresentadas por esses autores, o controle fuzzy ora melhorou o tempo de acomodação, ora reduziu o pico, mas não em todos os casos. Pode-se dizer em geral que o desempenho é próximo de um PID tradicional, às vezes um pouco melhor, às vezes pior.

Neste trabalho considerou-se o desenvolvimento de uma solução de malha fechada (Dorf and Bishop, 2009), devido à presença de sensores de deslocamento da posição relativa entre a carroceria e as rodas, que transmitem os dados ao controlador e que por sua vez atua no sistema mantendo essa posição nos limites definidos no projeto. Estudos com controladores difusos têm se ampliado, demonstrando sua viabilidade e equivalência com os controladores convencionais (Pontelli, 2012), (Crivellaro, 2008).

Com isto, o objetivo foi estudar e simular um sistema de controle ativo para suspensão automotiva usando lógica difusa (fuzzy logic), comparando-o com o controlador convencional proporcional e derivativo $(\mathrm{PD})$.

\section{MODELAGEM DO SISTEMA}

Ao analisar as oscilações do veículo, adotou-se os seguintes pontos de partida:

- O veículo está no movimento retilíneo a uma velocidade constante.

- As rodas estão sempre em contato com a estrada e esse é um contato de um ponto.

- A perturbação da estrada é a mesma na roda esquerda e direita e o carro é simétrico em relação ao eixo longitudinal.

No modelo da figura 1 , o corpo $1(m 1)$ é a parte da carroceria do veículo apoiada sobre uma roda e o corpo $2(\mathrm{~m} 2)$ refere-se ao pneu e roda e sua respectiva suspensão. Este é um modelo de dois sistemas massa-mola em paralelo, acoplados em série, o que justamente o difere dos modelos didáticos, que é um sistema simples massa-mola em série (Ogata, 2010). Neste modelo, os elementos são:
- m1 a massa da carroceria sobre a roda indicada,

- m2 a massa do conjunto roda, pneu e suspensão,

- k1 a mola e sua constante elástica,

- k2 o pneu e sua constante elástica,

- b1 a constante do amortecedor,

- b2 a constante de amortecimento do pneu,

- Fa representa a força do atuador para restringir a distância $X_{1}-X_{2}$ de oscilação entre a carroceria e a suspensão, e

- W o movimento dado pelas irregularidades da via.
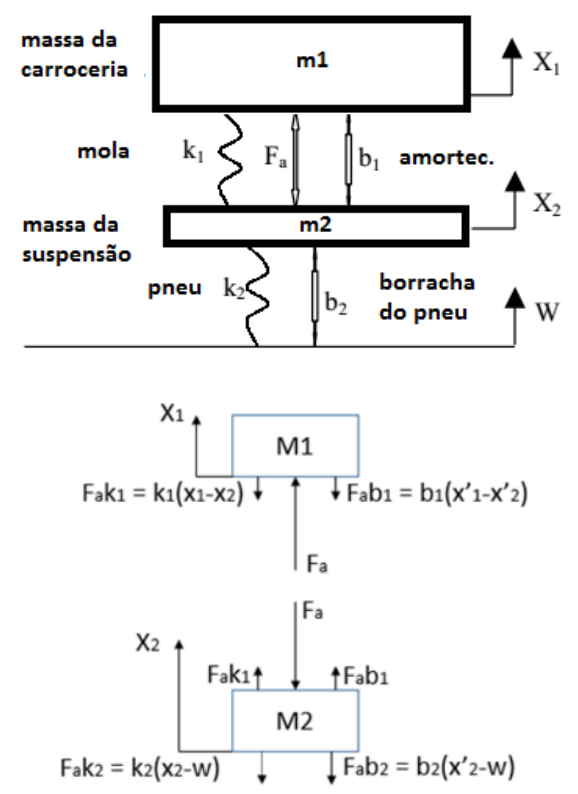

Figura 1. Modelagem das forças do sistema na suspensão

Sensores enviam dados ao sistema de controle sobre as posições $X_{1}$ e $X_{2}$. Quando o veículo está recebendo qualquer perturbação $W$ da via, o corpo 1 não deve ter grandes oscilações, as quais devem se dissipar rapidamente. Dado que a distância $X_{1}-W$ é muito difícil de medir e a deformação do pneu $X_{2}-W$ é desprezada, usou-se a distância $X_{1}-X_{2}$ em vez de $X_{1}-W$ como resultado nesse modelo. O distúrbio da via $W$ normalmente é simulado por um sinal de entrada tipo degrau. Esse sinal poderia representar o veículo que sai de um buraco com borda aguda. O objetivo é projetar um controlador para que a saída $X_{1}-X_{2}$ tenha um sobressinal (overshoot) inferior a $5 \%$ e um tempo de acomodação (settling time) menor que 5 segundos. Quando o veículo sobe em um degrau de $10 \mathrm{~cm}$ de altura, o corpo oscilará dentro de uma faixa de $5 \mathrm{~mm}$ e retornará a um percurso suave em até 5 segundos.

A partir da figura 1 e utilizando a $2^{\text {a }}$ lei de Newton, obtêmse as equações dinâmicas 1 e 2 :

$$
\begin{gathered}
m_{1} \ddot{X}_{1}=-b_{1}\left(\dot{X}_{1}-\dot{X}_{2}\right)-k_{1}\left(X_{1}-X_{2}\right)+U \\
m_{2} \ddot{X}_{2}=b_{1}\left(\dot{X}_{1}-\dot{X}_{2}\right)+k_{1}\left(X_{1}-X_{2}\right)+ \\
\quad+b_{2}\left(\dot{W}-\dot{X}_{2}\right)+K_{2}\left(W-X_{2}\right)-U
\end{gathered}
$$

Nestas equações, foi introduzida a ação $U$ do controlador, atuando fisicamente entre $m_{1}$ e $m_{2}$. A partir deste ponto, pode-se realizar uma abordagem que emprega a transformações de Laplace para se obter as funções de transferência. Neste trabalho, cujo objetivo é mostrar a 


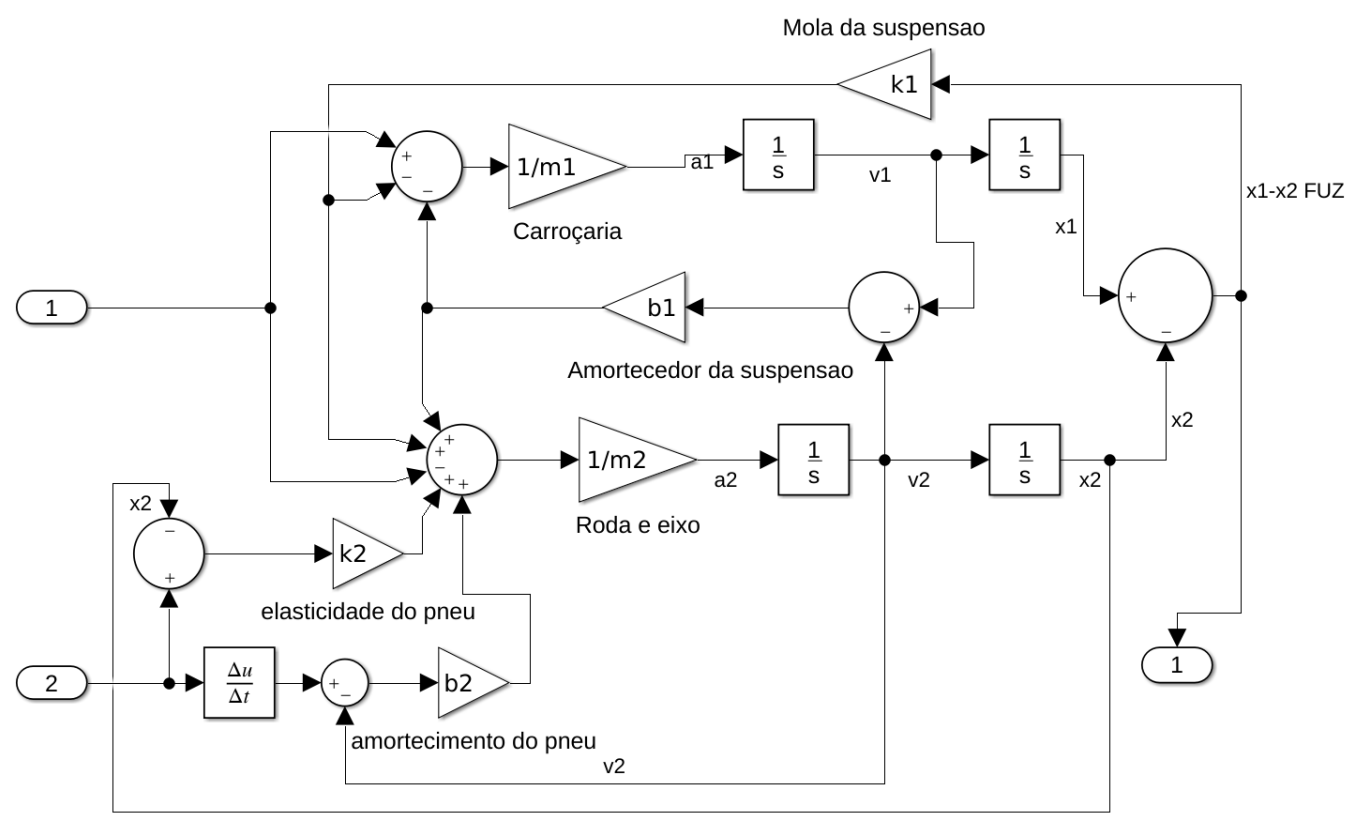

Figura 2. Modelagem da suspensão no Simulink



Figura 3. Modelagem dos sistemas de controle no Simulink. Os subsistemas são a suspensão da fig. 2

fase de projeto e testes em simulador, optou-se modelar o sistema através das equações dinâmicas 1 e 2 .

\subsection{Diagrama dos elementos físicos}

Este sistema foi modelado pela soma das forças atuando em ambas as massas (corpo e suspensão) e integrando as acelerações de cada massa para obter na primeira integração as velocidades e, na segunda, as posições (Messner and Tilbury, 2016) - ver equações 3 e 4 . O simulador empregado foi o Simulink, desenvolvido pela empresa MathWorks (MathWorks, 2014), que é um ambiente de programação gráfica para modelagem, simulação e análise de sistemas dinâmicos. Sua interface principal permite a diagramação de blocos gráficos, representando os diversos elementos básicos, contidos em uma biblioteca.

$$
\begin{aligned}
& \iint \frac{d^{2} x_{1}}{d t^{2}} d t=\int \frac{d x_{1}}{d t} d t=x_{1} \\
& \iint \frac{d^{2} x_{2}}{d t^{2}} d t=\int \frac{d x_{2}}{d t} d t=x_{2}
\end{aligned}
$$

O modelo dinâmico desenvolvido está na figura 2, que contém os seguintes blocos principais: 
- entradas 1 (do controlador) e 2 (da perturbação $W$ do solo);

- saída 1 - sinal do sistema controlado;

- valores das massas $\left(1 / m_{1}\right.$ e $\left.1 / m_{2}\right)$ de cada modelo massa-mola;

- valores das constantes elásticas da mola da suspensão $\left(k_{1}\right)$ e do pneu $\left(k_{2}\right)$;

- constantes de amortecimento da suspensão $\left(b_{1}\right)$ e do pneu $\left(b_{2}\right)$;

- integrais $1 / s$ no domínio de Laplace, convertendo acelerações $a_{i}$ em velocidades $v_{i}$ e estas em deslocamentos $x_{i}$;

- derivada da posição $x_{2}$ para compor o termo $b_{2} \cdot v_{2}$.

Os elementos da figura 2 recebem os sinais de perturbação do solo e de controle, que estão representados na figura 3 como subsistemas. Nesta figura, os blocos de interesse são os seguintes:

- fPD1 - controlador fuzzy desenvolvido neste trabalho;

- W - perturbação dada pelas irregularidades do solo;

- PD(s) - controlador PD utilizado para comparação;

- Subsystem 0, 1, 2 - modelo do sistema físico visto na figura 2.

\section{MODELAGEM DOS CONTROLADORES}

A modelagem de um controlador PID difuso é feita de modo a combinar os três sinais de controle proporcional, integrador e derivativo como em um PID convencional. Isto corresponde a gerar funções de pertinência em cada um dos três universos de discurso correspondentes. Na etapa seguinte, um conjunto de regras difusas de controle - fuzzy reasoning - devem ser projetadas de modo a reproduzir o comportamento de um controlador PID.

O modo de inferência fuzzy escolhido foi o de Mamdani. Para modelar e especificar sistemas fuzzy, pode-se por exemplo consultar Cox (1999). O número de regras geradas é igual ao produto cartesiano do número de funções de pertinência pelo número de grandezas (sinais) de controle. Se forem definidas cinco funções de pertinência por sinal de controle, são obtidas $5^{3}=125$ regras para serem associadas em combinadas de $N$ funções de pertinência no sinal de saída do controlador. Como a concepção destas regras e a modelagem e sintonia das funções de pertinência dependem de especialista externo, optou-se por um modelo de controle $P D$, encorajado pelo fato de que a constante integrativa de controle resultou em valores bem baixos, em simulações preliminares deste sistema com PID convencional.

O controlador difuso PD $(f P D)$ apresenta, então, dois sinais de entrada: proporcional ao erro (err) e proporcional à variação do erro - derivativo (derr), cujas regras difusas para o sistema de suspensão são vistas na tabela 1. A figura 4 (superior) mostra as funções de pertinência ajustadas para as grandezas err e derr na entrada e, na inferior, as funções para a saída $u$ do controlador. A escolha de sete funções de pertinência para cada parâmetro, quando poderiam ser menos delas, permite gerar transições mais suaves na superfície de controle, embora o esforço computacional seja maior. A construção das regras baseou-se nas premissas:
- se o erro é negativo e a variação é positiva (ou viceversa), então o sistema está retornando à posição de equilíbrio, logo a atuação deve ser mínima ou nula;

- se o erro e sua variação forem positivas (negativas), a ação de controle deve ser proporcionalmente positivas (negativas).

- demais regras tornam a superfície de controle suave.

Tabela 1. Regras fuzzy do controlador PD

\begin{tabular}{|c|c|c|c|c|c|c|c|}
\hline \multirow[b]{2}{*}{ err } & \multicolumn{7}{|c|}{ derr } \\
\hline & NB & NM & NS & $\mathrm{QZ}$ & PS & PM & PB \\
\hline NB & $\mathrm{NH}$ & $\mathrm{NH}$ & $\mathrm{NH}$ & NB & NM & NS & QZ \\
\hline NM & $\mathrm{NH}$ & $\mathrm{NH}$ & NB & NM & NS & $\mathrm{QZ}$ & PS \\
\hline NS & $\mathrm{NH}$ & NB & $\mathrm{NM}$ & NS & $\mathrm{QZ}$ & PS & $\mathrm{PM}$ \\
\hline $\mathrm{QZ}$ & NB & NM & NS & QZ & PS & $\mathrm{PM}$ & $\mathrm{PB}$ \\
\hline PS & NM & NS & $\mathrm{QZ}$ & PS & $\mathrm{PM}$ & PB & $\mathrm{PH}$ \\
\hline PM & NS & $\mathrm{QZ}$ & PS & $\mathrm{PM}$ & $\mathrm{PB}$ & $\mathrm{PH}$ & $\mathrm{PH}$ \\
\hline PB & $\mathrm{QZ}$ & PS & $\mathrm{PM}$ & PB & $\mathrm{PH}$ & $\mathrm{PH}$ & $\mathrm{PH}$ \\
\hline
\end{tabular}

1 NB (negative big), NM (negative medium), NS (negative small), QZ (quasi-zero), PS (positive small), PS (positive medium), PS (positive big), NH (negative huge), $\mathrm{PH}$ (positive huge)
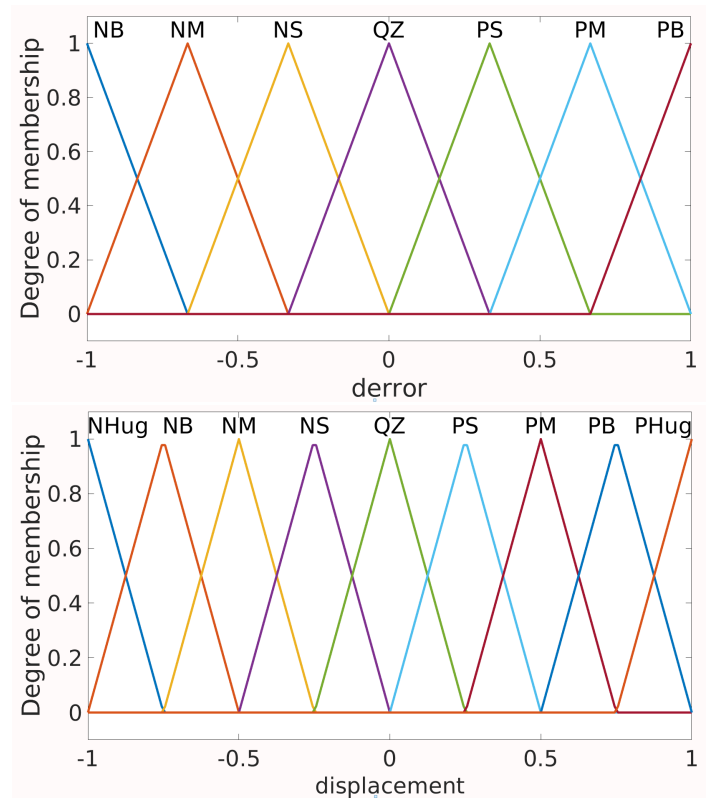

Figura 4. Funções de pertinência de entrada e saída

\section{Ajustes dos ganhos de entrada e saída}

Dado que controladores difusos são não-lineares, Jantzen (1998) propôs um procedimento para ajustar os ganhos de controladores fuzzy a partir de controladores PID convencionais. O ajuste aqui utilizado baseou-se nesse procedimento, adaptado para controlador fuzzy-PD $(f P D)$. Para que a sintonia ocorresse sistematicamente, todos os universos de discurso foram normalizados em [-1 1$]$ na entrada e na saída, conforme figura 4. Deste modo, tanto as regras difusas (tabela 1) quanto as funções de pertinência geram uma superfície de controle suave e simétrica. Deixando a sintonia do sistema apenas no ajuste dos ganhos, permite-se uma flexibilização tal que podem ser ajustados para cada sistema massa-mola amortecido em particular. A figura 3 destaca o controlador $f P D$ e os blocos de ganhos $G E$ (do erro), GDE (da variação do erro) e $G U$ (do deslocamento do atuador). 
O procedimento idealizado por Jantzen consiste basicamente em:

1 Calcular os parâmetros de sintonia de um controlador PID pelo método de Ziegler-Nichols de resposta em frequência (Dorf and Bishop, 2009) (Ogata, 2010).

2 Calcular os ganhos GE, GDE e GU do controlador $f P D$ (tabela 2 na $3^{\text {a }}$ linha) conforme parâmetros obtidos no item anterior.

Desta maneira, os requisitos de controle de cada sistema amortecido em particular podem ser definidos pelo procedimento acima, mantendo-se a generalidade das regras fuzzy de controle e a normalização do universo de discurso das grandezas de entrada e saída.

\section{RESULTADOS DAS SIMULAÇÕES}

Para o tipo de veículo escolhido (ônibus), foram adotados os seguintes valores sugeridos por Messner and Tilbury (2016)

\section{carroceria e suspensão}

$m_{1}=2500 \mathrm{~kg}$

$k_{1}=80000 \mathrm{~N} / \mathrm{m}$

$b_{1}=350 \mathrm{~N} . \mathrm{s} / \mathrm{m}$

$$
\begin{aligned}
& \text { roda e pneu } \\
& m_{2}=320 \mathrm{~kg} \\
& k_{2}=500000 \mathrm{~N} / \mathrm{m} \\
& b_{2}=15000 \mathrm{~N} . \mathrm{s} / \mathrm{m}
\end{aligned}
$$

\subsection{Estabilidade dinâmica do sistema}

Com os parâmetros acima, verifica-se que o sistema é estável pelo critério de Routh-Hurwitz (Dorf and Bishop, 2009), (Ogata, 2010). Obtendo-se a função de transferência a partir do sistema de equações 1 e 2 , os pólos $-33,3$ e 0 , e zeros $-2,65 \pm 13 j$ se encontram no semiplano esquerdo do diagrama root locus, demonstrando que o sistema é estável e subamortecido. Os cálculos foram realizados com o programa Matlab (Mathworks, 2015) pertencente a uma empresa na região do município. O gráfico do sistema da suspensão, sem ação de controle, é visto na figura 5. A carroceria do ônibus oscila por um tempo $>20$ s com amplitude inicial de $8 \mathrm{~cm}$. Não é uma oscilação confortável aos passageiros devido ao grande sobressinal e longo tempo de acomodação.

\subsection{Controle fuzzy-PD e convencional}

Para encontrar os valores dos parâmetros proporcional $k_{P}$ e derivativo $k_{D}$, pelo método de Ziegler Nichols, é necessário elevar $k_{P}$ até que se encontre uma oscilação sustentada, o qual se torna o parâmetro crítico $k_{C R}$. Obtém-se assim $k_{P}=0,6 k_{C R}$, levando ao período crítico de oscilação $P_{C R}$, de onde se obtém $T_{D}=0,125 P_{C R}$. Estes valores estão relacionados à tabela 2, que foram os seguintes: $k_{P}=832100, k_{D}=208000$ e $T_{D}=0,25 \mathrm{~s}$. O universo de discurso do erro está no intervalo [-1 1], por isso $G E=1,0$, resultando nos ganhos do controlador $f P D$ :

Tabela 2. Relações entre ganhos dos controladores convencional e fuzzy

\begin{tabular}{c|ccc} 
Controlador & $K_{P}$ & $1 / T_{I}$ & $T_{D}$ \\
\hline $\mathrm{fP}$ & $G E \cdot G U$ & & \\
$\mathrm{fINC}$ & $G D E \cdot G D U$ & $G E / G D E$ & \\
$\mathrm{fPD}$ & $G E \cdot G U$ & & $G D E / G E$ \\
$\mathrm{fPD}+\mathrm{I}$ & $G E \cdot G U$ & $G I E / G E$ & $G D E / G E$
\end{tabular}

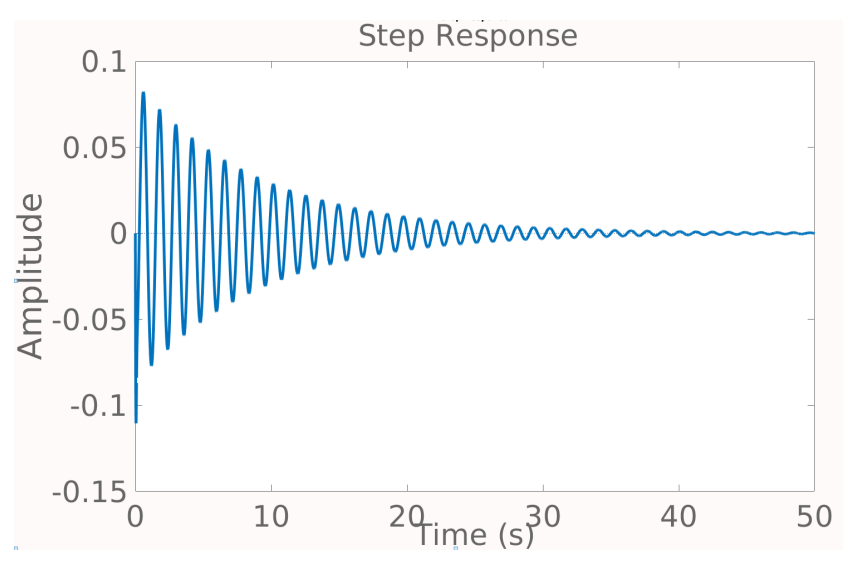

Figura 5. Resposta ao degrau de $10 \mathrm{~cm}$

$$
G D E=0,25 \quad \text { e } \quad G U=k_{P} / G E=832100
$$

Para a mesma perturbação de $10 \mathrm{~cm}$ para o caso nãocontrolado, o sistema apresentou uma oscilação inicial de $1 \mathrm{~cm}$, rapidamente anulada $(<1 \mathrm{~s})$, mas houve um offset residual de quase $-1 \mathrm{~cm}$. A solução proposta por Jantzen para eliminar isso foi somar a $G U$ (saída de controle) um ganho proporcional ao termo integrador, denominado de controle $f P D+I$ (tabela 2). No entanto, sem lançar mão desta solução, com a diminuição de $G U$ para cerca de 500000 , o offset reduziu para cerca de $3 \mathrm{~mm}$, aceitável em um projeto deste porte (figura 6). Observa-se que o sistema estabilizou em cerca de $2 \mathrm{~s}$ com um pico inicial de $-55 \mathrm{~mm}$ em resposta a um degrau de $-10 \mathrm{~cm}$.

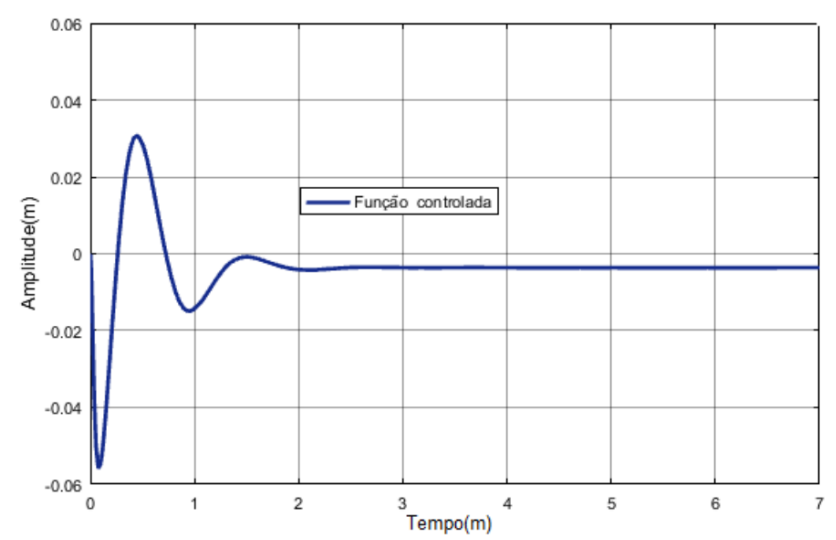

Figura 6. Resposta ao degrau de $-10 \mathrm{~cm}$ com controle fuzzy$\mathrm{PD}$

A figura 7 mostra o mesmo sistema com controlador PD convencional ajustado. Aqui também o sistema se acomoda em menos de 2 e o pico inicial $<-10 \mathrm{~mm}$ representa uma resposta melhor em termos de absorção do impacto inicial, porém ocorrem mais oscilações, introduzindo uma breve mas indesejável vibração no sistema.

\section{DISCUSSÃO E CONCLUSÕES}

Tanto o controlador PD tradicional quanto o fuzzy-PD forneceram condições suficientes para o controle da suspensão ativa automotiva apresentada neste trabalho. Fica claro que ainda é necessário melhorar o ajuste fino de ambos controladores, pois como foi visto, no sistema de suspensão 


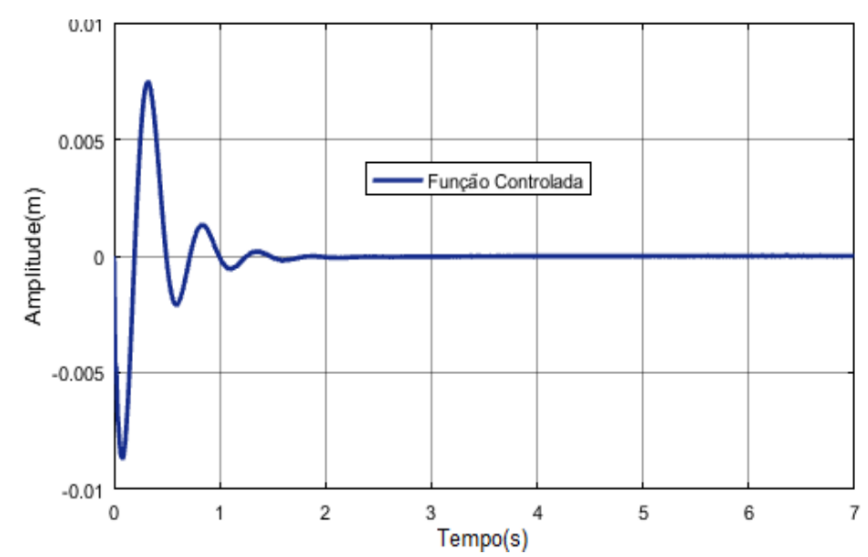

Figura 7. Resposta ao degrau de $-10 \mathrm{~cm}$ com controle PD convencional

ativa, seria necessário fazer um ajuste fino na saída do controlador FuzzyPD para melhorar o nível de referência. Ao contrário deste, no controlador PD tradicional o setpoint ficou dentro do especificado.

Embora um controlador puramente derivativo não seja implementável na prática, a ação derivativa, associada à ação proporcional, corresponde ao acréscimo de um zero ao sistema, atuando beneficamente no regime transitório, tendendo a aumentar a estabilidade relativa do sistema e reduzindo o tempo de acomodação. Contudo, ele aumenta o tempo de subida, e por não atuar no regime permanente, não corrige o erro de estado estacionário. Neste trabalho, esse problema foi minimizado com a introdução de um valor offset na saída do controlador.

A maior dificuldade encontrada para sintonizar esses dois controladores foi ajustar seus parâmetros, porque, além de envolver técnicas, experiência e conhecimento total do sistema a ser controlado, foram necessárias muitas simulações para atingir valores de sobressinal e acomodação razoáveis. O motivo disso é que o segundo método de Ziegler-Nichols não pode ser diretamente aplicado aos parâmetros do controlador Fuzzy, mas a solução proposta por Jantzen. A diretriz de projeto deste controlado fPD foi manter a normalização dos universos de discursos e a uniformidade na distribuição das funções de pertinência ao longo deles. Refinamentos na sintonia requerem alterações nesta distribuição e calibração nos limites dos universos de discurso, introduzindo muitos graus de liberdade (parâmetros ajustáveis) para a sintonia do controlador.

\section{Trabalhos futuros}

Por quê não foi utilizado um controlador PID? A adição do termo integrativo no controlador reduz o erro acumulado em vários ciclos. O equivalente fuzzy de um controlador PID implica em adicionar mais uma entrada no bloco $f P D 1$ da figura 3 e, portanto, a inclusão de um universo de discurso com algumas funções de pertinência. Se for mantida a resolução de sete funções de pertinência, haverá $7^{3}$ regras de inferência, cujo ajuste requer uma metodologia alternativa à de Jantzen, gerando uma superfície de controle mais complicada. Este é uma abordagem a ser investigada em um trabalho posterior.
O ajuste alcançado com fPD foi bastante satisfatório ao reduzir em um o número de oscilações em relação ao controlador PD.

\section{REFERÊNCIAS}

Al-Mutar, W.H. and Abdalla, T.Y. (2015). Quarter car active suspension system control using fuzzy controller tuned by pso. International Journal of Computer Applications, $127(2)$, 38-43.

Changizi, N. and Rouhani, M. (2011). Comparing pid and fuzzy logic control a quarter car suspension system. The Journal of Mathematics and Computer Science, 2(3), 559-564.

Corriga, G., Sanna, S., and Usai, G. (1991). An optimal tandem active-passive suspension system for road vehicles with minimum power consumption. IEEE Transactions on industrial eletronics, 38(3).

Cox, E. (1999). The fuzzy systems handbook: a practitioner's guide to building, using, and maintaining fuzzy systems. AP Professional, 2 edition. URL https:// books.google.com.br/books?id=2udQAAAAMAAJ.

Crivellaro, C. (2008). Controle robusto de suspensão semi-ativa para caminhonetes utilizando amortecedores magneto-reológicos. Dissertação de mestrado, Escola Politécnica da Universidade de São Paulo, São Paulo.

Daimler (2016). Active Body Control $A B C$. http://techcenter.mercedesbenz.com/en/abc/detail.html. Access jun 152016 .

Dorf, R.C. and Bishop, R.H. (2009). Sistemas de controle modernos. LTC, Rio de Janeiro.

Halliday, Resnick, and Walker (2002). Física, volume 1. LTC, Rio de Janeiro.

Jantzen, J. (1998). Tuning of fuzzy pid controllers. techreport 98-H 871, Technical University of Denmark.

Mathworks (2015). Matlab. URL. URL https://www . mathworks. com/products/matlab.html.

Messner, B. and Tilbury, D. (2016). Control tutorials for matlab \& simulink. techreport, Michigan University, http://ctms.engin.umich.edu/CTMS/index.php?example=Susp

Mulla, A.A. and Unaume, D.R. (2013). Active suspensions future trend of automotive suspensions. In International Conference on Emerging Trends in Technology \& its Applications, ICETTA 2013.

Ogata, K. (2010). Engenharia de controle moderno. Pearson, 5 edition.

Pontelli, C.O. (2012). Estratégias de controle para isolação ativa de vibrações em barras de pulverizadores agrícolas. Dissertação de mestrado, Universidade de São Paulo, Escola de Engenharia de São Carlos, São Carlos.

Popovic, V., Jankovic, D., and Vasic, B. (2000). Design and simulation of active suspension system by using matlab. In Seoul 2000 FISITA World Automotive Congress, 1-8.

Senthilkumar, P., Sivakumar, K., Kanagarajan, R., and Kuberan, S. (2018). Fuzzy control of active suspension system using full car model. Mechanika, 24(2), 240-247.

Soleymani, M.M. (2010). Investigation of the energy regeneration of active suspension system in hybrid electric vehicles. IEEE TRANSACTIONS ON INDUSTRIAL ELECTRONICS, 57(3), 918-925.

Strassberger, M. and Guldner, J. (2004). Bmw's dynamic drive: an active stabilizer bar system. IEEE Control Syst. Mag, 28-29. 